\title{
Lightness, brightness, and brightness contrast: 2. Reflectance variation
}

\author{
LAWRENCE E. AREND \\ Eye Research Institute, Boston, Massachusetts \\ and \\ BRANKA SPEHAR \\ Rutgers University, Newark, New Jersey
}

\begin{abstract}
Changes of annulus luminance in traditional disk-and-annulus patterns can be perceived to be either reflectance or illuminance changes. In the present experiments, we examined the effect of varying annulus reflectance. In Experiment 1, we placed test and standard patch-and-surround patterns in identical Mondrian patchworks. Only the luminance of the test surround changed from trial to trial, appearing as reflectance variation under constant illumination. Lightness matches were identical to brightness matches, as expected. In Experiment 2, we used only the patch and surround (no Mondrian). Instructions said that the illumination would change from trial to trial. Lightness and brightness-contrast data were identical; illumination gradients were indistinguishable from reflectance gradients. In Experiment 3, the patterns were the same, but the instructions said that the shade of gray of the test surround would change from trial to trial. Lightness matches were identical to brightness matches, again confirming the ambiguity of diskand-annulus patterns.
\end{abstract}

The experimental paradigm introduced in the first paper of this series (Arend \& Spehar, 1993) lets us measure three distinct attributes of the perception of a neutral (achromatic) scene. Conditions were established under which the three tasks produced very different quantitative data for exactly the same physical stimuli. Armed with the paradigm and baseline data from that paper, we are now ready to take a new look at lightness matches in classical disk-and-annulus patterns.

Our new stimuli allow us to make a significant advance over most previous work on the influence of background reflectance on lightness and brightness. Both the lightness and the brightness of a gray patch are affected by the reflectance of the immediately surrounding area (Arend \& Spehar, 1993; Gilchrist, Delman, \& Jacobsen, 1983; Koffka, 1935; Takasaki, 1966; Whittle, 1992; Whittle \& Challands, 1969). A piece of gray paper on a white background paper appears both darker gray and dimmer (less bright) than a piece of the same paper on a black background. Most previous investigators have used traditional disk-and-annulus stimuli. With these stimuli, variation of the luminance of the annulus as the indepen-

We thank Alan L. Gilchrist for making Branka Spehar's visit possible and for valuable discussions. Paul Whittle and three anonymous reviewers provided numerous helpful suggestions. This research was supported by Air Force Office of Scientific Research Grants AFOSR 86-0128 and AFOSR 89-0377 to L.E.A. B.S. was supported by NSF Grant BNS-8909182 to A. L. Gilchrist. Correspondence should be addressed to L. E. Arend, David Sarnoff Research Center, CN 5300, Princeton, NJ 08543-5300. dent variable is perceived to consist either of changes in the reflectance of the test annulus or of changes in the illuminance of the test disk-and-annulus array, depending on the instructions to the subject.' (See Figure 1.)

The Mondrian region of our stimuli allows less ambiguous manipulation of these two parameters. The test patch and surround tend to appear to be under the same illumination as the surrounding Mondrian. If only the surround luminance is changed and its luminances all lie within the fixed luminance range of the Mondrian, they appear to be changes of the reflectance of the surround. Conversely, simultaneous proportional changes of the luminances of all the Mondrian patches and the surround compellingly appear to be changes in the illumination falling on the entire test-patch-surround-Mondrian array.

In the preceding study (Arend \& Spehar, 1993), this separation of illumination and reflectance variation allowed us to vary, independently, both the illumination on the test patch and the reflectance of its surroundings. One benefit was that we were able to examine, for the first time, both the deviation from lightness constancy produced by a difference of surround reflectances and the dependence of that error on illumination level.

In the present paper, we report three experiments that extend our new paradigm to variation of surround reflectance and, at the same time, clarify the meaning of earlier research with ambiguous disk-and-annulus patterns.

In Experiment 1, we used the patch-and-surround-inMondrian paradigm to vary the surface color (reflectance) of the test surround. Our observers adjusted the test patch to match the standard in lightness, brightness, and local 


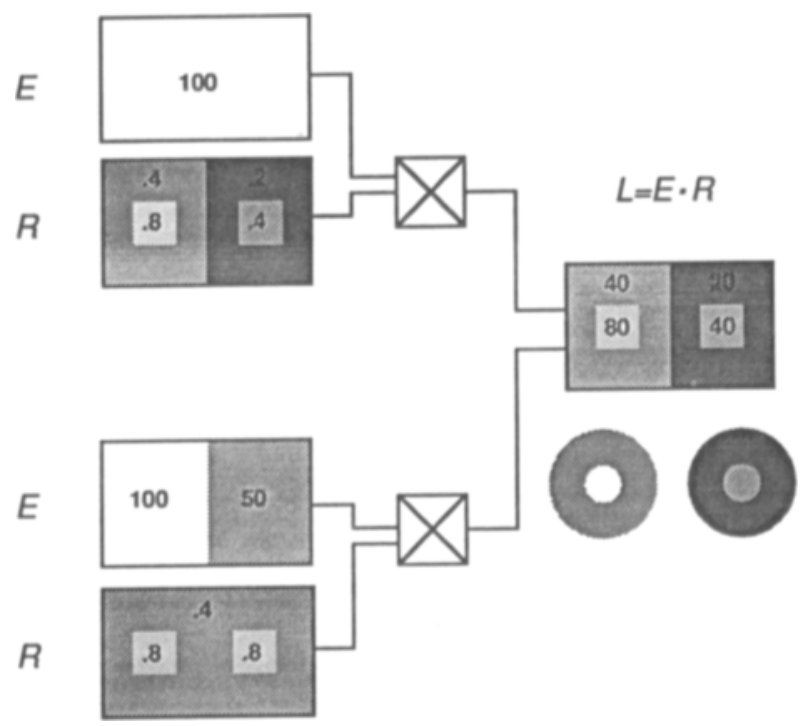

Figure 1. Disk-and-annulus patterns are perceptually ambiguous. The pattern of retinal illuminances is as likely to be due to two illuminations falling on a homogenous background with a single reflectance as to a single illumination falling on two backgrounds with different reflectances.

brightness contrast. The brightness and brightness-contrast matches resembled those from the illumination-variation experiments in our previous paper. The lightness matches, on the other hand, were very different. The lightness matches closely resembled the brightness matches, as was expected, given the apparently equal and constant illuminations of the test and standard patterns. Thus, the context of the Mondrian dramatically altered the apparent surface color (lightness) without changing the brightness or brightness contrast. This indicates that the Mondrian affected the perceptual meaning of the local luminance contrast information rather than the local information itself, confirming and extending Gilchrist et al.'s (1983) results.

In Experiments 2 and 3, we used identical stimuli, the ambiguous classical patch-and-surround patterns, without the Mondrian surrounds. In Experiment 2, the observers were told that the illumination on the right-hand (test) patch and surround would vary from trial to trial. Under these conditions, the changes of surround luminance were perceived to be illumination changes on a constant reflectance. ${ }^{2}$ Thus, the patch and surround had roughly the same appearance as the (photometrically identical) patch-and-surround portion of the stimuli in our preceding study (Arend \& Spehar, 1993). The brightness and brightness-contrast matches resembled those that we used before, but the ambiguity of the patch and surround produced very different lightness matches. Without the Mondrian, the observers have no way of knowing that part of the luminance difference between the luminances of the test and standard annuli is due to different reflectances. They attribute all of the difference to illumination. As a consequence, when they try to match lightnesses, they actually match brightness contrasts.

In Experiment 3, the stimuli were identical to those of Experiment 2, but the instructions were changed. The observers were now instructed to consider the illuminations on the standard and test arrays to be fixed and equal, and they were told that the shade of the gray surface (reflectance) of the surround surrounding the test patch would change from trial to trial. Thus, the patch and surround had roughly the same appearance as they did in Experiment 1. As in Experiment 1, the brightness matches resembled those in the illumination-variation experiments (Arend \& Spehar, 1993; the present Experiment 2), but the lightness matches were dramatically different. The lightness matches were nearly identical to the brightness matches. Thus, the perceptual meaning of local brightness contrasts is defined by global context when the stimulus is rich enough (Arend \& Spehar, 1993; the present Experiment 1) but by observer expectations when the stimulus is ambiguous (Experiments 2 and 3 ).

\section{EXPERIMENT 1 Reflectance Variation in Mondrians}

In our initial experiments (Arend \& Spehar, 1993), we simulated variation from trial to trial of the illuminance on the entire test Mondrian. The luminances of all the patches in the Mondrian of the test array were therefore scaled up and down as required by the changing illuminance. Lightness invariance under those conditions constitutes classical lightness constancy.

Now we want to simulate a situation in which the reflectance of the surround around the test patch changes from trial to trial while the illumination of the test array is constant and equal to that on the standard array. Lightness invariance under these new conditions has sometimes been called "Type 2" constancy or "constancy over backgrounds."

\section{Method}

Stimuli. Details of the apparatus and calibrations can be found in Arend and Spehar (1993).

The stimuli were computer simulations of uniformly illuminated matte papers lying in a common depth plane; they were presented on a carefully calibrated color monitor. For brevity we will hereafter drop the simulation terminology and refer to the stimuli and their simulated properties as though they were actual papers.

The stimuli (Figure 2) were similar to those in the illuminancevariation experiment (Arend \& Spehar, 1993). The test and standard arrays consisted of a central patch centered in two rectilinear surround regions. The inner surround (hereafter called the surround) was uniform in luminance. The outer surround (hereafter called the Mondrian) was a patchwork of simulated reflectances.

For this experiment, only the luminance of the inner surround changed from trial to trial. The luminances in the Mondrian of the test array were held constant and equal to those in the standard array. As a result, the illuminance on the entire test array appeared to be constant, and the apparent reflectance of the surround around the test patch varied from trial to trial.

Five test-surround luminances were used, those produced by the combinations of illuminance and reflectance in the illuminancevariation experiment (Arend \& Spehar, 1993, Figure 2). The 19:1 


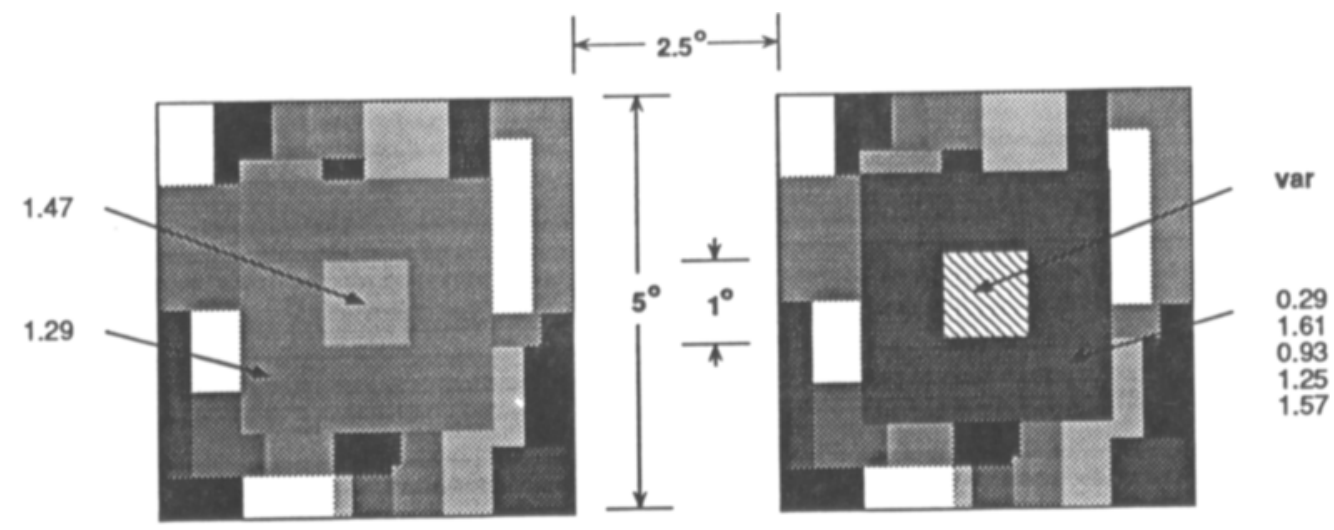

a.
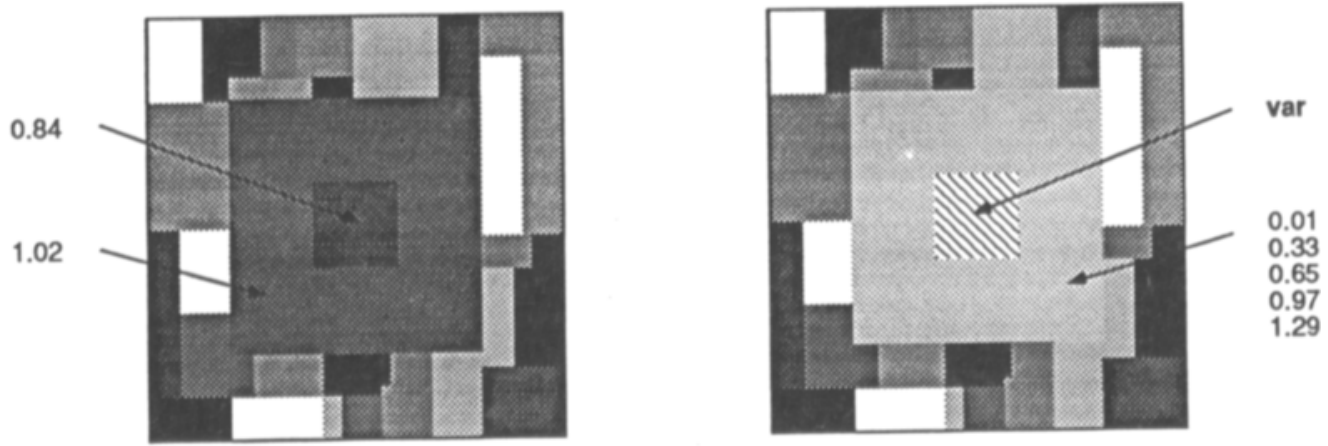

b.

STANDARD

TEST

Figure 2. Diagrams of patch-and-surround-in-Mondrian stimulus patterns. Reflectances were identical in Mondrian portions of test (right) and standard (left) arrays, ranging from 0.03 to 0.95 . Illuminances on the test and standard arrays were fixed and equal. Values shown are $\log$ luminances $\left(\mathrm{cd} / \mathrm{m}^{2}\right)$. Luminance (simulated reflectance) of the test surround varied from trial to trial over a 19:1 range. The subjects varied the luminance of the test patch to match the standard patch by one of three criteria. (a) increment condition. (b) decrement condition.

luminance range of the surround corresponded to reflectances ranging from 0.04 to 0.76 , appearing black and white, respectively.

Procedure. The procedures were the same as in Arend and Spehar (1993). The subjects initially adapted for 3 min to a large gray field of the average luminance of the test and standard Mondrians. They then viewed the two continuously presented displays and matched the test patch in the right display to the corresponding standard patch in the left display, using the mouse of a graphics tablet to adjust the test-patch luminance. The match was made to satisfy one of three task criteria, described below. The subjects were asked to spend about the same amount of time looking at the test and standard patterns and to alternate their gaze between the patterns, shifting approximately once per $2 \mathrm{sec}$.

Tasks. The three tasks (brightness, lightness, brightness contrast) were also the same as in Arend and Spehar (1993).

In the lightness match condition, we instructed the subjects to make the test patch "look as if it were cut from the same piece of paper" as was the corresponding patch in the standard.

In the brightness match condition, we instructed the subjects to make the test patch "have the same brightness as the corresponding patch in the standard, disregarding, as much as possible, other areas of the display. That is, make the amount of light coming from the test patch look the same as that from the standard."
In the brightness contrast task, we instructed the subjects to "make the brightness difference between the test patch and surround the same as that between the standard patch and surround."

Subjects. Three observers participated: the authors (L.A. and B.S.) and a paid observer (D.A.). B.S. was familiar with the purpose of the experiment but had no prior experience in lightness or brightness matching. D.A. was experienced in lightness and brightness matching but naive with respect to the purpose of the experiment.

\section{Results}

Lightness, brightness, and brightness-contrast matches are shown in Figure 3 as circles, squares, and triangles, respectively, with the observers' mean log luminance settings plotted as a function of the luminance of the test surround. The horizontal solid lines are the loci of test-patch luminances that equal the standard-patch luminances. The slanted solid lines are the luminances for which the patch/ surround luminance ratio in the test array matched that in the standard array.

For increments, the lightness and brightness matches were nearly identical, as would be expected given that 

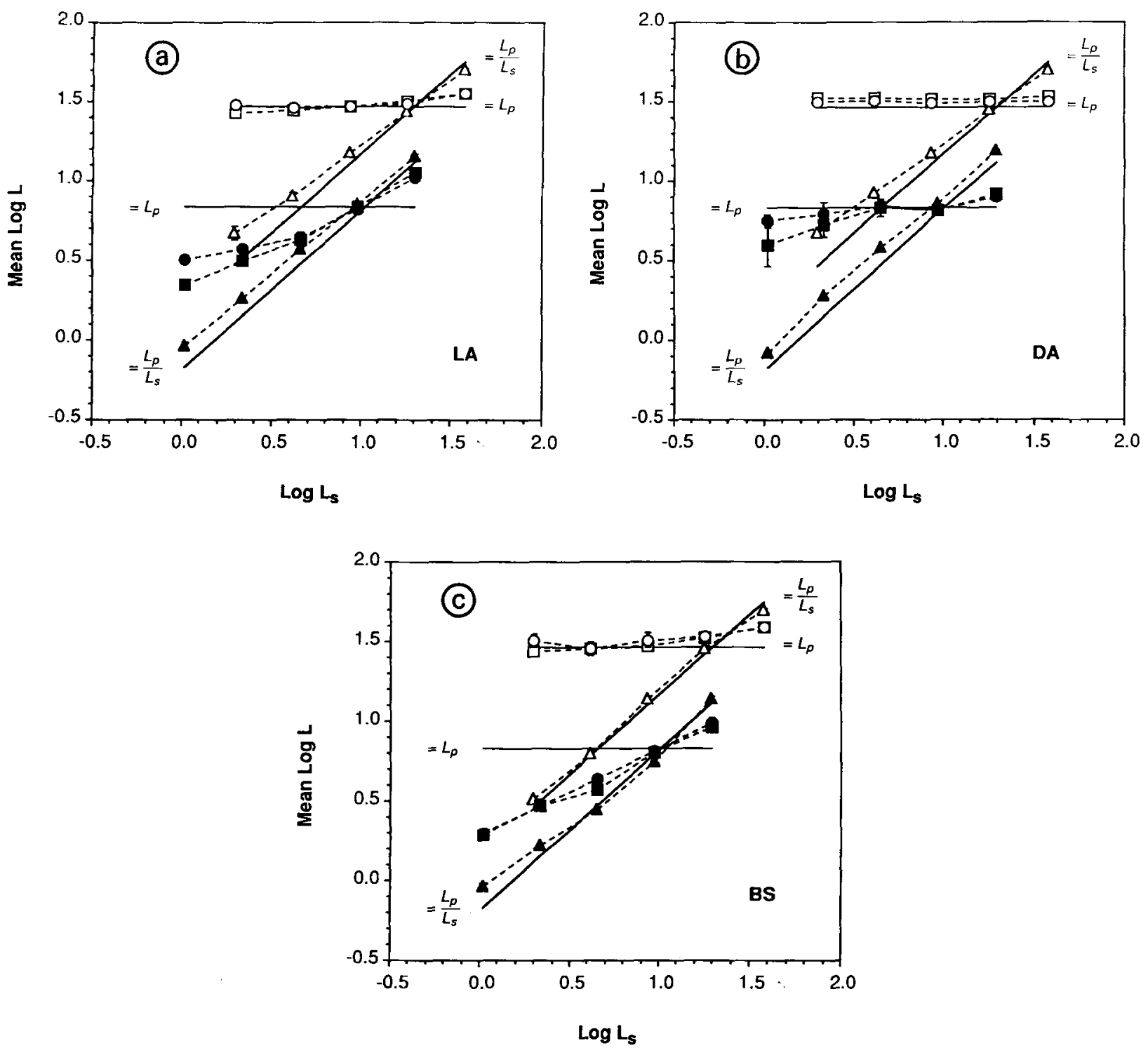

Figure 3. Mean log luminance data for patch-and-surround-in-Mondrian stimulus. Reflectance of the surround in the test array varied from trial to trial. The illumination on both test and standard arrays was constant and equal. Solid lines: theoretical lines, explained in text. Circles: lightness matches. Squares: brightness matches. Triangles: brightness-contrast matches. Open symbols: increments. Filled symbols: decrements. All points have error bars. Error bars are $\pm 1 S E(n=5)$. If no bar is visible, $\pm 1 S E$ is smaller than the plot symbol. (a) Observer L.A. (b) Observer D.A. (c) Observer B.S.

the illuminations of the test and standard appeared to be equal. For both lightness and brightness, all 3 observers produced nearly perfect luminance matches for the increments. Under these experimental conditions, that constitutes perfect invariance of lightness. When the test and standard are under the same illumination, equal reflectances have equal luminances. Thus, changing the surround luminance did not affect lightness, and there was no failure of lightness invariance due to local simultaneous contrast effects.

These increment data also strongly contradict Wallach's $(1948,1976)$ hypothesis: lightness matches did not require equal local luminance ratios.
For the decrements, on the other hand, the data did follow a pattern describing a local simultaneous contrast effect. As the surround reflectance increased from its minimum, the observer had to increase the luminance of the test patch to maintain constant brightness and lightness. When the luminance of the test surround equaled that of the standard surround (where the solid lines cross), the observers set perfect luminance matches, and when the test-surround luminance exceeded that of the standard surround, test-patch luminance had to exceed the luminance match. For 2 observers, the local simultaneous contrast effect may have been slightly larger for brightness than for lightness at the lowest surround luminances. 
The decrement data also clearly contradict Wallach's hypothesis. The local simultaneous contrast effect was in the right direction but not large enough to place the lightness matches on the equal-ratio theoretical line.

The brightness-contrast data followed the same pattern as in the illuminance-variation experiment (Arend \& Spehar, 1993). They approximated luminance-ratio matches for all 3 subjects. The increment data for L.A. and D.A. departed from the theoretical line in the same way as in Figure 3 of our preceding study; that is, greater luminance contrast was required at the lower background luminances. This failure of Weber's law reduces slightly the large difference in the slopes of the brightness-contrastand lightness-matching curves, but they remain well separated.

\section{EXPERIMENT 2 \\ Illuminance Variation, Patch/Surround}

Now that we have measured lightness, brightness, and brightness contrast in patterns with perceptually unambiguous illumination (Arend \& Spehar, 1993) and reflectance (the present Experiment 1) changes on the test patch and surround, we have a firm basis from which to evaluate the classical disk-and-annulus paradigm.

In general, we would argue (as in the introduction) that patterns as ambiguous as the disk and annulus should not be used in studies of surface appearance, because of problems of understanding the meaning of the data with respect to normal perception. We include them here only to provide a concrete illustration of the relationship between our experiments with less ambiguous patterns and the large past literature on disk-and-annulus patterns.

At this point, we must pause to make a crucial distinction. With patterns as ambiguous as the patch and surround, the instructions to the subject are critically important, and the argument in our previous paper regarding the effects of instructions does not apply. In this case, the perception is multistable, and the change of instructions does result in a change of the perceptual response. The pattern actually looks different when the variation of the surround luminance is perceived as reflectance changes. The change of appearance due to instructions is of the same variety as that in the reversal of the Necker cube or the girl and crone-a change of interpretation of the sensory data.

On the other hand, when the outer Mondrian ring was present, the relationship between the surround luminance and the luminances in the Mondrian controlled the appearance. In our preceding study (Arend \& Spehar, 1993), when the luminances of the Mondrian were scaled up and down by the same factor as the variation of the surround luminance, both the Mondrian and the surround were perceived to be undergoing identical illumination changes. With that stimulus, instructions that describe the surround changes as reflectance changes would be merely confusing, because the observer is unable to ignore the correlation between the Mondrian changes and the surround changes. The Mondrian changes are unambiguously perceived to be illumination changes. When, however, the Mondrian luminances were held constant over trials while the luminance of the surround was changed (Experiment 1 ), the surround was readily perceived to have different gray values (reflectance) from trial to trial. That configuration is not quite as unambiguous as when the Mondrian and surround covary. It is difficult but possible to perceive the surround changes to be illumination changes. One can perceive, for example, that the patch and surround lie in a more distant plane, viewed through a hole cut in the Mondrian.

\section{Method}

The stimuli were square patch-and-surround patterns approximating the classical disk-and-annulus patterns (Figure 4). Two $1^{\circ}$-square patches were presented $7.5^{\circ}$ apart (center to center), each surrounded by a gray, square surround whose inner border coincided with the edge of the patch and whose outer border subtended $5^{\circ}$. Thus, the overall geometry was as that in our first study (Arend \& Spehar, 1993), but with the Mondrian surround replaced by the enlarged uniform surround.

The procedures were the same as in the previous study (Arend \& Spehar, 1993) in all other respects. The luminances were the same as in the patch-and-surround portion of the Mondrians in our previous simulation of illumination changes (Arend \& Spehar, 1993, Figure 1). The luminances of the standard array were fixed. The luminance of the surround of the test array varied from trial to trial, in five steps over a 19:1 range.

The observers were told that the illumination on the right-hand (test) patch and surround would vary from trial to trial. They performed the lightness, brightness, and brightness-contrast matching tasks of Experiment 1, in separate sessions.

\section{Results}

The matches are plotted in Figure 5, with the same coordinates as for the Mondrian experiments in Arend and Spehar (1993). The illumination level on the test array

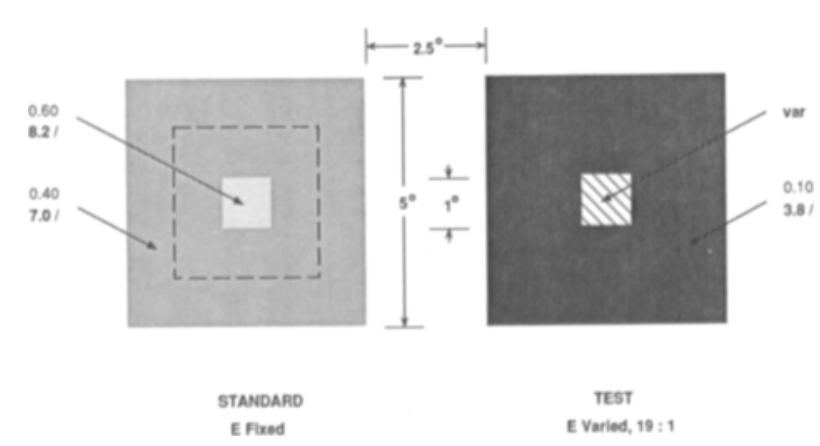

Figure 4. Diagram of patch-and-surround stimulus patterns. Plain type: reflectance. Boldface: equivalent Munsell value. Values are shown only for the increment condition. The decrement condition values are as in the patch-and-surround patches of Arend and Spehar (1993, Figure 1). Mondrian portions of Figure 2 test (right) and standard (left) arrays were replaced by an enlarged, uniform surround. The illuminance on the standard array was fixed. The illuminance on the test array varied from trial to trial, in five steps over a 19:1 range. The subjects varied the luminance of the test patch to match the standard patch according to one of three criteria. 
(a)

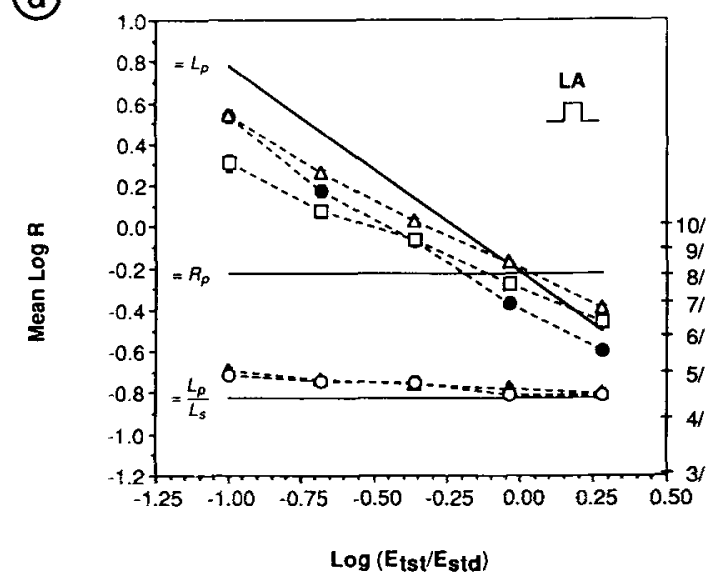

(b)

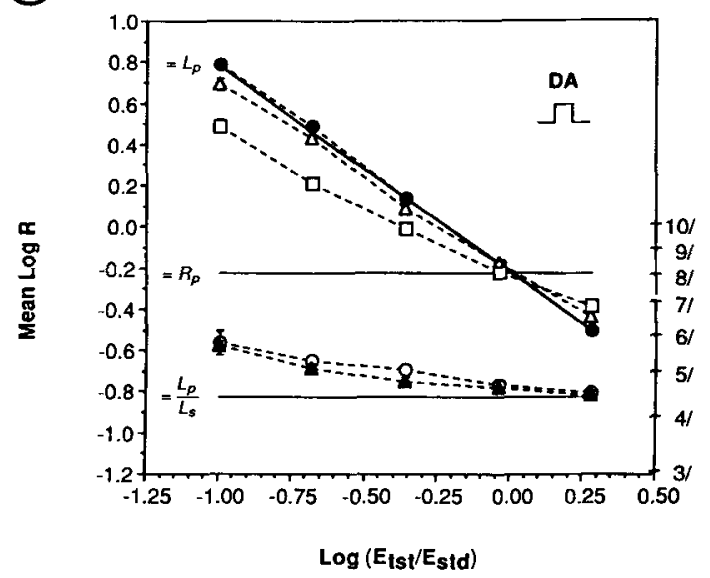

(c)

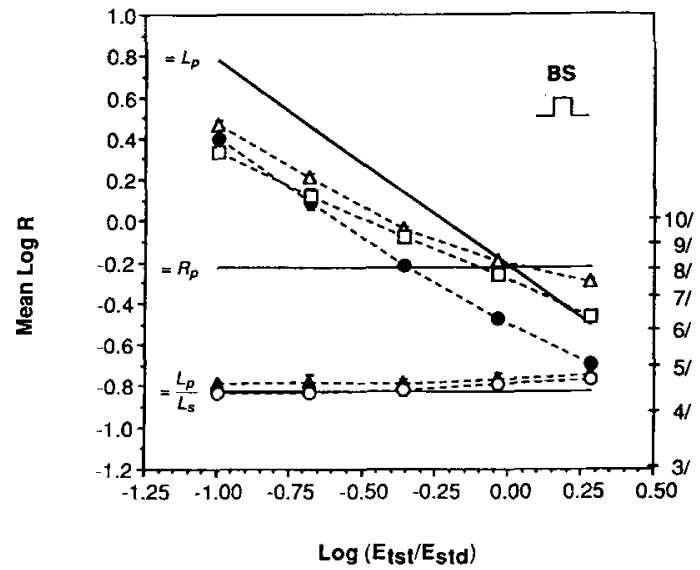

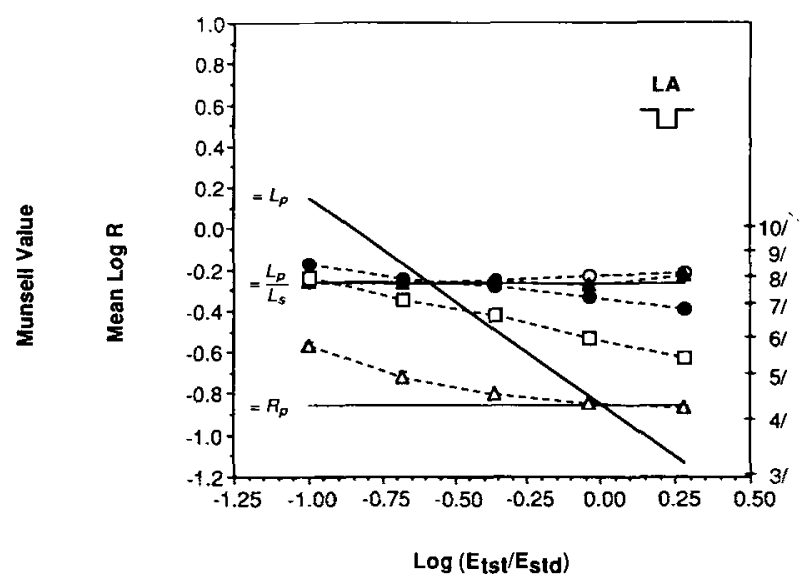

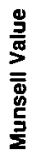

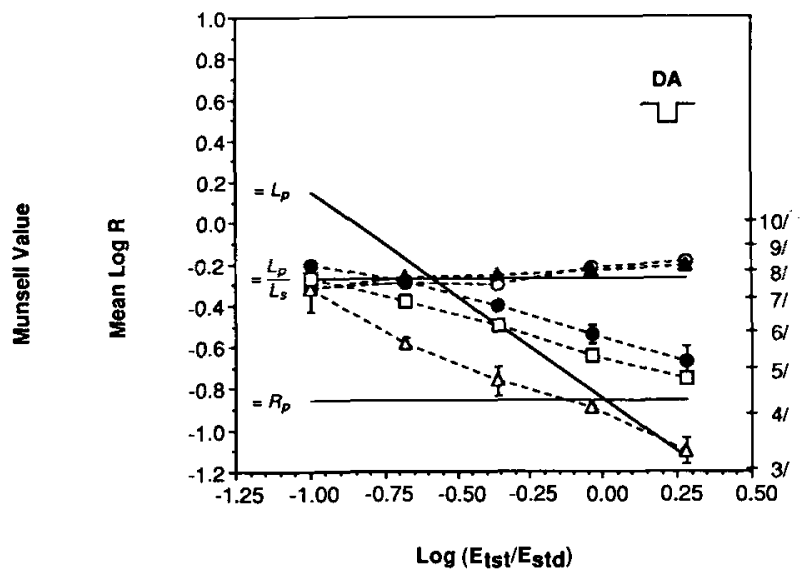

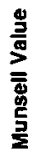

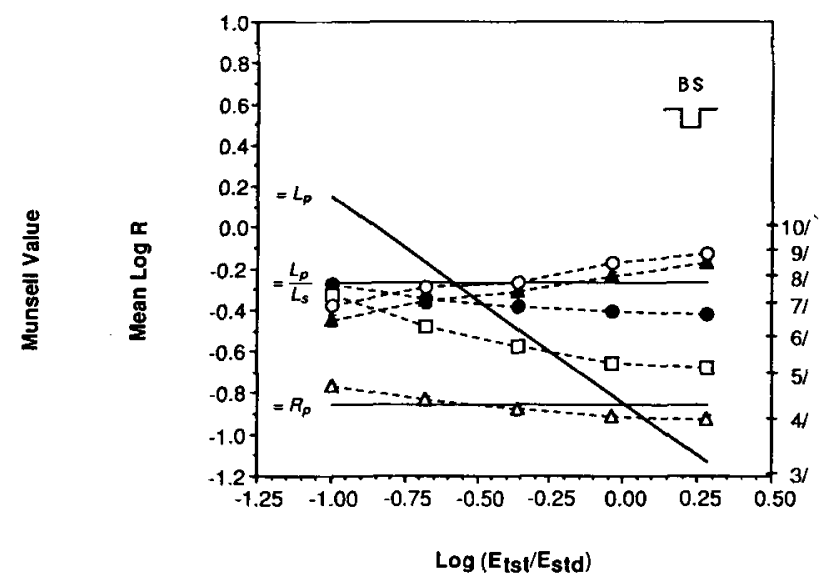

Figure 5. Mean log reflectance data for patch-and-surround stimulus. Left panels: increments. Right panels: decrements. Solid lines: theoretical, explained in text. Open circles: lightness matches. Filled circles: brightness matches. Filled triangles: brightness-contrast matches. Open squares and open triangles: comparison brightness data (explained in text). All points have error bars. Error bars are $\pm 1 S E(n=5)$. If no bar is visible, $\pm 1 S E$ is smaller than the plot symbol. (a) Observer L.A. (b) Observer D.A. (c) Observer B.S. 
is indicated on the horizontal axis by the log of the ratio of the illuminance of the test array to the fixed illuminance of the standard array. The subjects' mean log reflectance settings (mean log luminance minus log illuminance) are plotted as ordinates. For comparison, the Munsell values corresponding to the log reflectances of the left vertical axis are indicated on the right vertical axis.

The theoretical lines are also the same, but they have new interpretations when the Mondrian is not present.

Lightness and brightness contrast. The top horizontal solid line in the increment condition (Figure 5, left panels) and the bottom horizontal solid line in the decrement condition (Figure 5, right panels) are at the simulated reflectance of the standard patch-they are the theoretical perfect lightness-constancy lines. The other horizontal lines are at the reflectance that gives the same patch/surround luminance ratio in the test and standard arrays.

The open circles and filled triangles in each panel are the means for lightness and brightness-contrast matches, respectively.

The most important feature of the data is that the lightness matches are identical to the brightness contrast matches from this experiment and the corresponding patch-and-surround-in-Mondrian experiment (Arend \& Spehar, 1993). They lie near the equal-luminance-ratio theoretical line rather than the equal-reflectance line, and the increment data have the negative slope characteristic of the brightness-contrast matches. In the experiment with Mondrians, on the other hand, lightness constancy was nearly perfect.

The reader may object that the equal-reflectance theoretical lines are meaningless in this experiment. That is precisely our point about the perceptual ambiguity of diskand-annulus stimuli (Figure 1). Our computer program treated the test surround as having a different reflectance from that of the standard surround, and the illumination was calculated exactly as before. However, the physical model embodied in our simulation is completely arbitrary without the Mondrian. The subjects had no way of knowing that the annuli had different reflectances. They were told that the illumination on the test patch and surround would vary from trial to trial. It is true that the percept under these conditions is stable and systematic. The annuli appear to have equal reflectances: all the brightness difference between the two annuli is attributed to illumination differences. However, this is true no matter what reflectances and illuminances the experimenter uses to produce the stimuli. If adequate care is taken to eliminate artifacts, this is as true for real surfaces and lights as it is for simulations. The ambiguity of disk-and-annulus displays is a logical, not an empirical, fact.

With disk-and-annulus patterns, when observers are told to make lightness matches they can only match brightness contrasts. With disk-and-annulus patterns, there are only two aspects of neutral color perception available to be matched, brightness and brightness contrast.

Brightness. Brightness matches were obtained by using two different sets of test-surround luminances. In one
(Figure 5, filled circles), the luminances were the same as for the lightness and local simultaneous contrast tasks, the luminances required by our simulation of test and standard annuli with different reflectances. For purposes of comparison, we also ran a second condition (open triangles), in which the simulated reflectance of the test surround was the same as that of the standard surround. Since there was no Mondrian, this means simply that each luminance of the test surround was shifted by a factor of four, the ratio of the test and standard surround reflectances in the unequal-reflectance condition.

The matches for equal surround reflectances (open triangles) provide a reference for evaluation of the shift due to local simultaneous contrast when the surround reflectances were unequal (filled circles). For decrements, the shift ranged from approximately 2 Munsell value steps for D.A. to nearly 3 steps for B.S. For increments, it was approximately 2 steps for L.A. and B.S. As in other conditions, D.A. showed little simultaneous contrast effect for increments.

These reference data also provide a crude idea of factors other than the local simultaneous contrast effect. The open-triangle curve at the 0.0 abscissa is the brightness match for identical test and standard annuli, so shifting the filled-circle curve vertically to that point is one way to compensate for the local simultaneous contrast effect. The increment data would then lie on the equal-luminance line, ${ }^{3}$ representing photometric matches (D.A. produced nearly perfect photometric matches without this shift). For decrements, as in previous experiments (Arend \& Goldstein, 1987, 1990), the slope was substantially shallower than that of the equal-luminance line .

The open squares are the patch-and-surround-in-Mondrian brightness data from the first paper of this series (Arend \& Spehar, 1993), replotted here to allow comparison of the stimulus patterns. All conditions were identical except for the replacement of the Mondrian with the wider uniform surround. The most obvious difference is the smaller local simultaneous contrast effect for decrements in the Mondrian condition. The magnitude of the effect is easiest to see at the 0.0 abscissa, the point at which the test and annulus luminances were equal. For L.A. and B.S., the effect with the Mondrian was approximately 1 Munsell value step rather than 2 or 2.5 with the uniform surround. It is unlikely that areal summation can account for the difference between the stimulus patterns. The $1^{\circ}$ surround of the Mondrian pattern is already near the upper asymptote for areal summation found in previous studies of brightness induction.

\section{EXPERIMENT 3 \\ Reflectance Variation, Patch/Surround}

In Experiment 2, the observers were told that the illumination would change from trial to trial. With the ambiguous patch and surround and those instructions, the variation from trial to trial of the luminance of the test surround appeared to result from illumination change. On the other hand, they could instead be told that the illumi- 
nations were fixed and that the reflectance of the test surround would change from trial to trial. Under these instructions, the experiment is no longer a study of lightness invariance under illumination change. Rather, it is a study of lightness invariance under change of background reflectance, as in Experiment 1.

\section{Method}

The stimulus patterns had the same geometry as in Experiment 2 (Figure 4), the patch and surround without Mondrian. The luminance of the test surround varied over the same five values as in Experiment 1 (reflectance variation, patch and surround with Mondrian). The observers were now instructed to consider the illuminations on the standard and test arrays to be fixed and equal, and they were told that the shade of the gray surface (reflectance) of the surround surrounding the test patch would change from trial to trial. They performed the same lightness task as in Experiment 1. The brightness and brightness-contrast tasks were omitted, because, with these patterns and instructions, they were nearly identical to those in Experiment 2. All other procedures were unchanged.

(a)

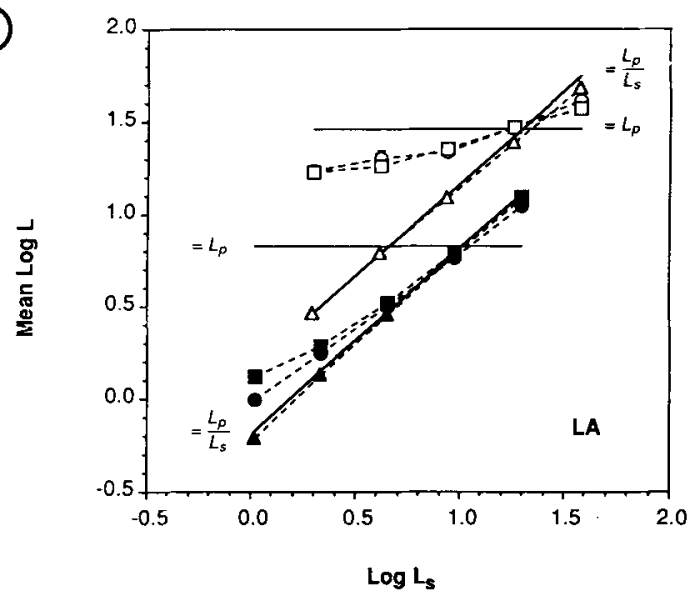

\section{Results}

Lightness matches under the reflectance-change instructions are shown as open circles in Figure 6, with mean $\log$ luminance plotted against the log of the test-surround luminance. The horizontal solid lines are the loci of test-patch luminances that equal the standard-patch luminances. The slanted solid lines are the luminances for which the patch/surround luminance ratio in the test array matched that in the standard array.

The lightness matches were approximately the same as the brightness matches (squares) from Experiment 2, as would be expected given that the illuminations of the test and standard appeared to be equal. For comparison, the lightness matches (triangles) from the first experiment with patch-and-surround-in-Mondrian stimuli (Arend \& Spehar, 1993, Figure 2) are also plotted.

For increments, D.A. produced nearly perfect luminance matches, just as when the Mondrian was present
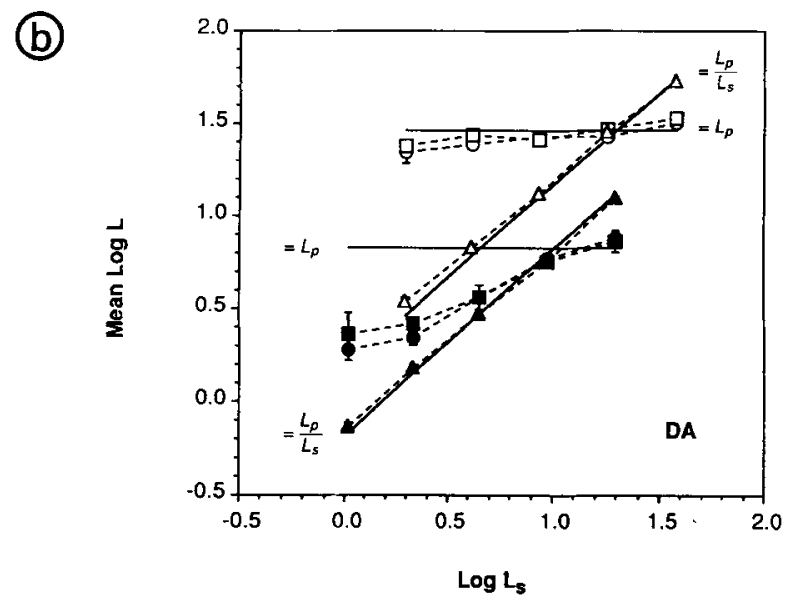

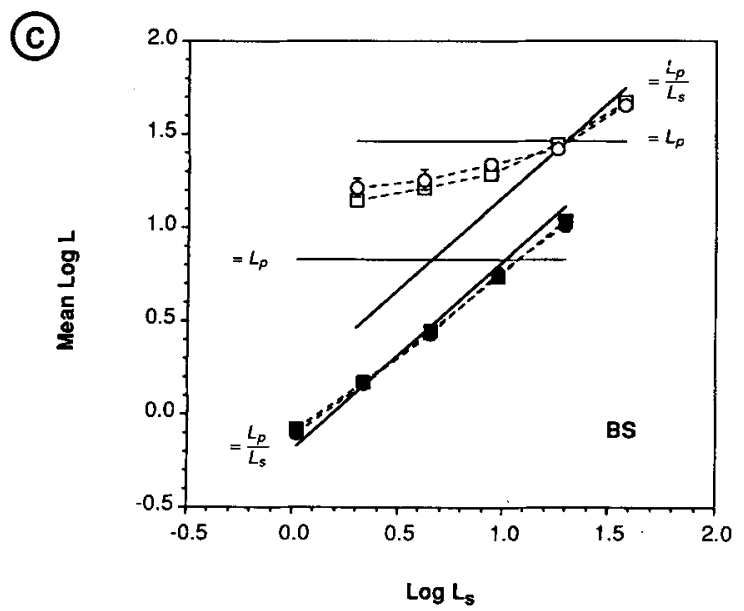

Figure 6. Mean log luminance data for patch-and-surround stimulus. The subject was instructed that reflectance of the surround in the test array would vary from trial to trial. Solid lines: theoretical lines, explained in text. Circles: lightness matches. Squares: brightness matches from Figure 5. Triangles: lightness matches from Arend and Spehar (1993, Figure 2; not available for Observer B.S.). Open symbols: increments. Filled symbols: decrements. All points have error bars. Error bars are $\pm 1 S E(n=5)$. If no bar is visible, $\pm 1 S E$ is smaller than the plot symbol. (a) Observer L.A. (b) Observer D.A. (c) Observer B.S. 
(Experiment 1). For him, there was no effect of changing the surround luminance-no local simultaneous contrast effect. For L.A. and B.S., there was a slight local simultaneous contrast effect for increments, 0.2 log units, when the test-surround luminance was a log unit less than that of the standard surround. The presence of the surround in Experiment 1 somehow prevented the local simultaneous contrast effect.

This local simultaneous contrast effect was far too small to place the lightness matches on the equal-ratio theoretical line. As in Experiment 1, the increment data strongly contradict Wallach's hypothesis: lightness matches did not require equal local luminance ratios.

For the decrements, on the other hand, the data showed a strong local simultaneous contrast effect. For L.A. and B.S., the lightness data were almost on the equal-ratio theoretical line. For D.A., the effect was weaker. While this provides limited agreement with Wallach's hypothesis, it is important to note that it does so at the expense of lightness constancy. In the present Experiment 3, the test and standard illuminances were equal, so lightness constancy was equivalent to luminance matches. For B.S., for example, there was a large (1 log unit) departure from lightness constancy when the test-surround luminance was a $\log$ unit less than that of the standard surround.

Although these data demonstrate that the observers could consistently follow the instructions in our patch-andsurround experiment, they should not be interpreted as supporting the use of disk-and-annulus stimuli in studies of apparent surface color. It is still not possible to understand the results from disk-and-annulus experiments in which the instructions less clearly restricted the observers' interpretation of the perceptually multistable stimulus. It is preferable to provide less ambiguous stimuli (like our Mondrians) rather than rely on instructions to clarify an ambiguous display.

\section{DISCUSSION}

\section{Perceptual Interpretation of Local Brightness Contrast}

The experiments in this paper complete our study of perception of local brightness contrast and its dependence on more global structure.

Wallach was right about the importance of local luminance ratios, but he was wrong about how that information is used in surface perception. As Gilchrist et al. (1983) elegantly demonstrated, a local brightness contrast, the response correlate of a local luminance ratio, is ambiguous with respect to its physical causes. In our simulations here of matte, coplanar scenes, local brightness contrast is due to some combination of surface reflectance gradients and illumination gradients. Global context can produce confident, stable perception of apparently changing illumination and fixed surface lightness (Arend \&
Spehar, 1993) or vice versa (Experiment 1). In more general scenes with three-dimensional structure and specular variation, there are many more possible physical and perceptual possibilities, but there too, rich global context typically allows a reliable, stable perception of the physical scene. The processes by which the visual system computes such percepts are complex and largely unknown. Arend (in press-b) has reviewed recent perceptual research related to those processes and suggested a preliminary, heuristic model, based on concepts from computational vision.

When the global context is too impoverished (patch and surround only), the changes of brightness contrast can be perceived to be either a varying reflectance under fixed illumination (Experiment 2) or vice versa (Experiment 3), depending on the observer's attitude or set. In this situation, the percept is modal: any particular change of the surround luminance is perceived to be either a reflectance change or an illumination change, but not both simultaneously.

Local brightness contrast itself was little affected by whether the outer surround was uniform or Mondrian (Figure 7). There were no substantial differences between the two conditions for Subjects L.A. and D.A. Whereas Subject B.S. required significantly greater physical contrasts for increments with the Mondrian surround (Figure 7 , open squares), for decrements there was a small difference in the opposite direction.

\section{Arend and Goldstein's (1987) Patch/Surround Condition}

Arend and Goldstein's (1987) interpretation of their patch-and-surround lightness data was incorrect. The data lay approximately along theoretical equal-reflectance lines. As in the case of the data for their Mondrian stimuli, they interpreted that as good lightness constancy. In the case of the patch-and-surround stimuli, however, that interpretation was entirely arbitrary. The experimental program generated a luminance for the test surround on each trial. Without the Mondrian, there is no connection of that luminance with a unique combination of illumination and reflectance. The particular reflectance and illuminance represented by the theoretical line was entirely in the mind of the experimenters. The theoretical line for the "black" stimulus could just as logically have been replaced by the theoretical line for the "white" stimulus (and the illuminance ratios on the abscissas shifted by a factor of 0.03 to reflect the corresponding decrease of theoretical illuminance). The data would then have been described as showing no lightness constancy. Those data, like the data reported here, were brightness-contrast matches, not lightness matches. Their patch-and-surround "lightness" data even showed the slight slopes characteristic of our brightness contrast matches, whereas their Mondrian lightness data, like our lightness data, had slopes of approximately zero. 
(a)

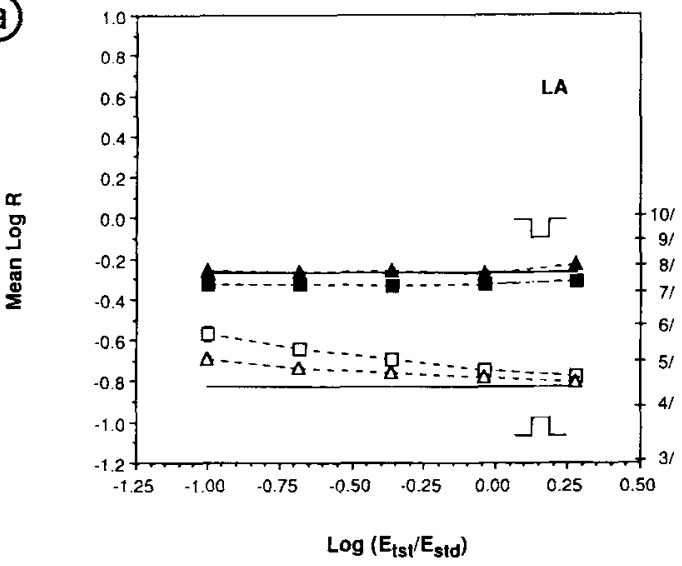

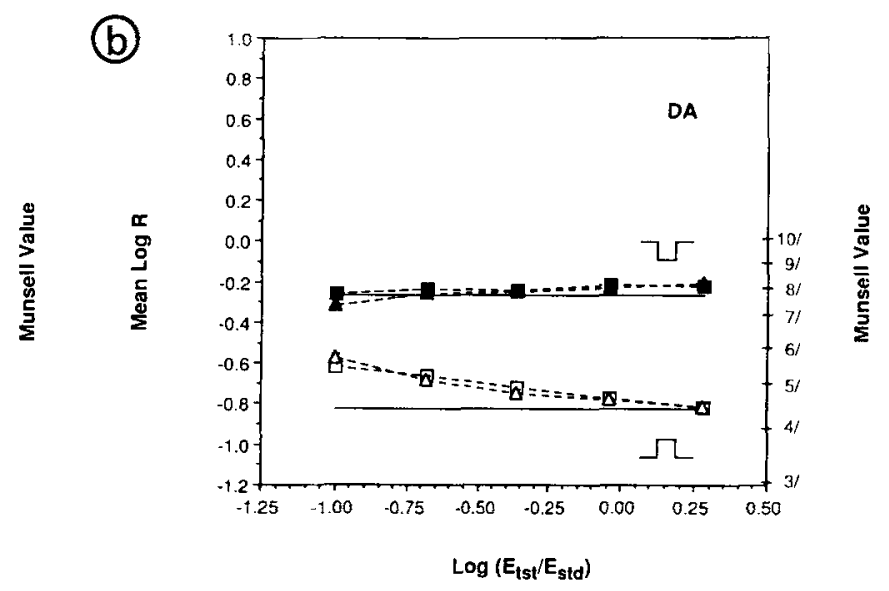

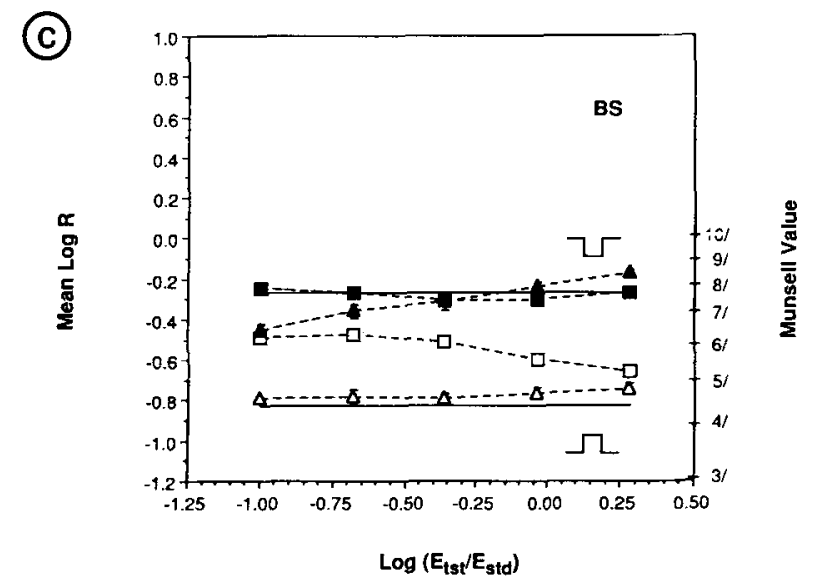

Figure 7. Comparison of brightness-contrast data for patch-and-surround stimulus with and without Mondrian region. Open symbols: increments. Filled symbols: decrements. Solid lines: theoretical, explained in text. Triangles: brightness-contrast matches without Mondrian region (same as filled triangles, Figure 5). Squares: brightness-contrast matches with Mondrian region (same as triangles in Figure 3 of Arend \& Spehar, 1993). All points have error bars. Error bars are $\pm 1 S E(n=5)$. If no bar is visible, $\pm 1 S E$ is smaller than the plot symbol. (a) Observer L.A. (b) Observer D.A. (c) Observer B.S.

\section{Effect of Surround Structure on Magnitude of Simultaneous Contrast}

The influence of the several different types of surround in our experiments on the magnitude of local simultaneous contrast can be assessed by comparing brightness matches from conditions in which the inner, uniform surrounds are the same. Three such conditions are plotted in Figure 8 . The square symbols are brightness matches from Experiment 1 (Figure 3), in which the outer surround was a fixed-luminance Mondrian. The circles are from the illumination-variation experiment of Arend and Spehar (1993, Figure 2), in which the outer surround was a Mondrian, the luminances of which covaried with the luminance of the inner surround. The triangles are from Experiment 2 (Figure 5), in which the outer surround was uniform, with luminances equal to those of the inner surround.

For each of the three types of surround, the form of the brightness data in Figure 8 can be readily understood as a consequence of larger local simultaneous contrast effects for decrements than for increments. When the test and standard surround luminances are equal (where the theoretical lines cross), the brightness match requires equal luminances of the test and standard patches. As the luminance of the test surround decreases, the luminance of the test disk required for a brightness match steadily declines because of a decrease in local simultaneous contrast. This is true for both increments and decrements, but the stronger local simultaneous contrast influence for decrements than for increments produces a faster decline for the decrements. The difference between the slopes of the brightness curves for increments and decrements is thus simply a second manifestation of the asymmetry of the local simultaneous contrast effect that was responsible for the different offsets of the increment and decrement curves in Figure 3 of Arend and Spehar (1993).

The different slopes of the three pairs of curves in Figure 8 are somewhat surprising. Classical studies of simul- 
(a)

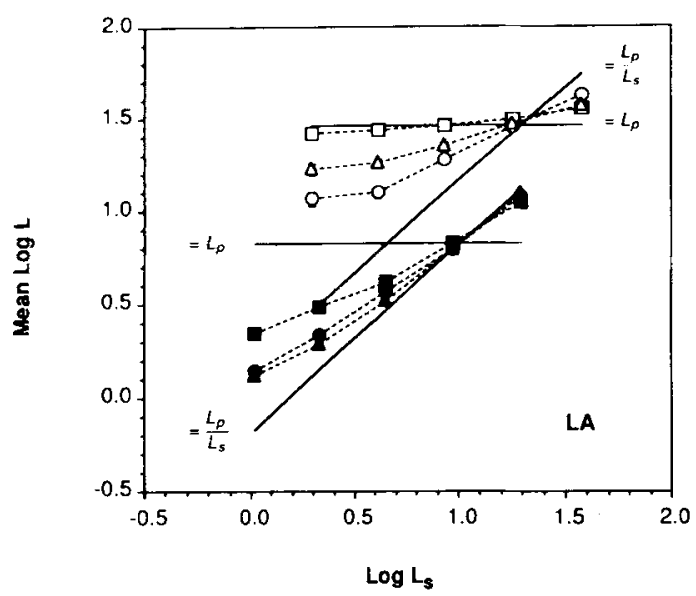

(b)

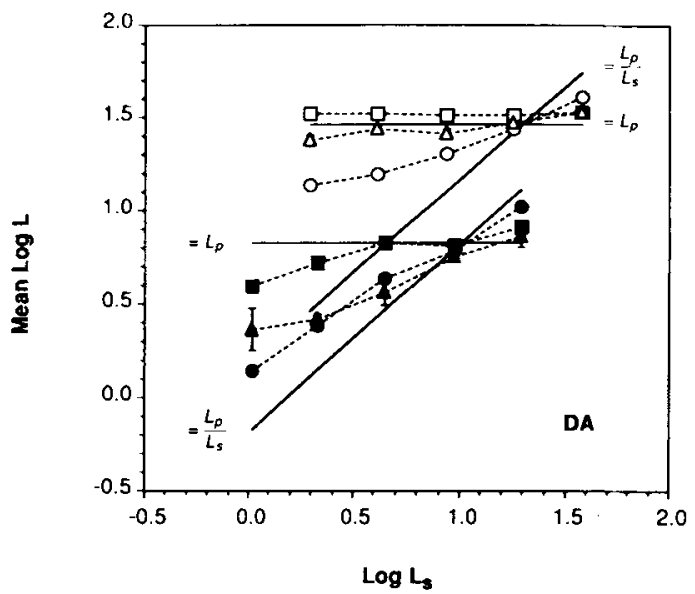

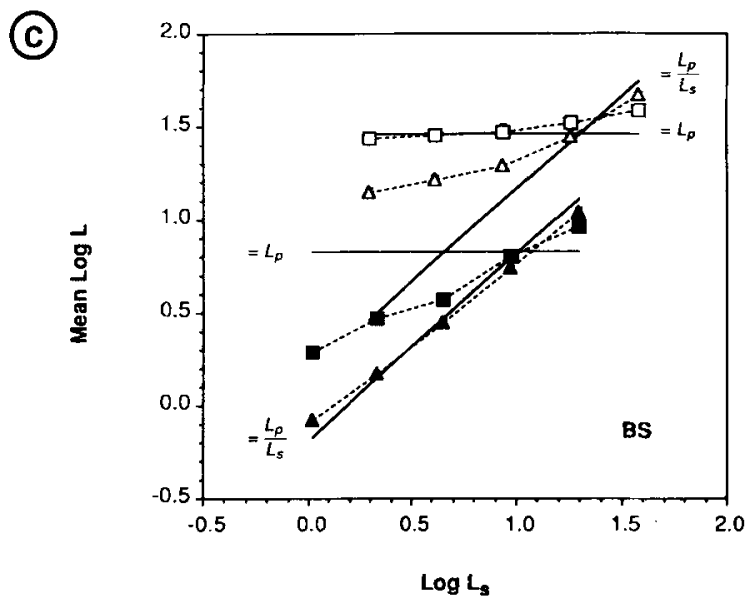

Figure 8. Brightness match data from three surround conditions. Surround luminance varies identically; the outer surround is different for the three conditions. Solid lines: theoretical lines, explained in text. Squares: outer surround fixed-luminance Mondrian (Experiment 1, Figure 3). Circles: outer surround Mondrian; luminances covary with inner surround (Arend \& Spehar, 1993, Figure 2). Triangles: outer surround uniform, luminance equal to inner surround (Experiment 2, Figure 5). Open symbols: increments. Filled symbols: decrements. All points have error bars. Error bars are $\pm 1 S E(n=5)$. If no bar is visible, $\pm 1 S E$ is smaller than the plot symbol. (a) $O b-$ server L.A. (b) Observer D.A. (c) Observer B.S. (did not participate in the circle condition).

taneous brightness contrast have typically found little effect for inducing fields that are more than $1^{\circ}$ of visual angle from the test field (see, e.g., Brown \& Mueller, 1965; Leibowitz, Mote, \& Thurlow, 1953). Since all of our stimulus patterns were identical within the central patch and $1^{\circ}$ of visual angle inner surround, one might expect no differences in brightness matches among the several outer-surround configurations.

In the simulation of illuminance changes in the inner surround (Figure 8, circles), both the luminance of the inner surround and that of the outer Mondrian surround were varied. In the simulation of reflectance changes in the inner surround (Figure 8, squares), only the luminance of the inner surround varied. The luminances of the outer Mondrian surround were fixed. The shallower slopes of the curves with squares than of the curves with circles in Figure 8 indicates that the outer surround affected the brightness of the test patch, despite the $1^{\circ}$ of visual angle separation.

Slightly larger departures from luminance matching occurred when the Mondrian ring (Figure 8, circles) replaced the uniform ring (Figure 8, triangles). By the preceding account, this would imply slightly stronger local simultaneous contrast effects when the outer ring had high luminance variance.

\section{REFERENCES}

AREND, L. E. (1990). Mesopic lightness, brightness, and brightness contrast. In OSA annual meeting technical digest 1990 (p. 206). Washington, DC: Optical Society of America. (Abstract)

AREND, L. E. (in press-a). Mesopic brightness, lightness, and brightness contrast. Perception \& Psychophysics. 
AREND, L. E. (in press-b). Surface colors, illumination, and surface geometry: Intrinsic-image models of human color perception. In A. L. Gilchrist (Ed.), Lightness, brightness, and transparency. Hillsdale, NJ: Erlbaum.

AREND, L. E., \& Goldstern, R. (1987). Simultaneous constancy, lightness and brightness. Journal of the Optical Society of America A, 4, 2281-2285.

AREND, L. E., \& GoldsteIN, R. (1990). Lightness and brightness over spatial illumination gradients. Joumal of the Optical Society of America A, 7, 1929-1936.

AREND, L. E., \& SPEHAR, B. (1993). Lightness, brightness, and brightness contrast: 1. Ulluminance variation. Perception \& Psychophysics, 54, 446-456.

Brown, J., \& Mueller, C. (1965). Brightness discrimination and brightness contrast. In C. H. Graham (Ed.), Vision and visual perception (pp. 208-250). New York: Wiley.

Evans, R. M. (1974). The perception of color. New York: Wiley.

GiLCHRIST, A. L. (1988). Lightness contrast and failures of constancy: A common explanation. Perception \& Psychophysics, 43, 415-424.

Gilchrist, A. [L.], Delman, S., \& Jacobsen, A. (1983). The classification and integration of edges as critical to the perception of reflectance and illumination. Perception \& Psychophysics, 33, 425-436.

HoCHBERG, J. (1986). Representation of motion and space in video and cinematic displays. In K. R. Boff, L. Kaufman, \& J. P. Thomas (Eds.), Handbook of perception and human performance (chap. 9, pp. 1-57). New York: Wiley.

KoFFKA, K. (1935). Principles of Gestalt psychology. New York: Harcourt, Brace, \& World.

Leibowitz, H., Mote, F. A., \& Thurlow, W. R. (1953). Simultaneous contrast as a function of separation between test and inducing fields. Journal of Experimental Psychology, 46, 453-456.

Rock, 1. (1975). An introduction to perception. New York: Macmillan.

TAKASAKI, H. (1966). Lightness change of grays induced by change in reflectance of gray background. Journal of the Optical Society of America, 56, 504-509.

WALLACH, H. (1948). Brightness constancy and the nature of achromatic colors. Journal of Experimental Psychology, 38, 310-324.

WALLACH, H. (1976). On perception. New York: Quadrangle/The New York Times Book Co.

WhitTle, P. (1992). Brightness, discriminability and the "Crispening Effect." Vision Research, 32, 1493-1507.
Whittle, P., \& Challands, P. D. C. (1969). The effect of background luminance on the brightness of flashes. Vision Research, 9, 1095-1110.

\section{NOTES}

1. It is not completely clear at this point how successful this manipulation can be in various situations. Complete experimental control requires that the display be confined to just the four fields (two disks, two annuli) in a perfectly dark surround, with microtexture and other "extraneous" stimulus properties eliminated. Only a few experimenters have exercised such complete experimental control. If these precautions are carefully followed, the stimulus is a perfect simulation of either physical arrangement, and the observer's expectations (usually determined by instructions) should determine which is perceived. It has been noted by several authors (e.g., Evans, 1974; Rock, 1975; Wallach, 1948) that the appearance of the annulus of a bright disk and annulus in a dark surround is intermediate between the luminous quality of a bright disk surrounded by darkness and the surface quality of a disk surrounded by a more luminous annulus. It has also been argued, however, that stimuli this simple always remain somewhat ambiguous, with instructions merely biasing the judgments one way or the other (Gilchrist, 1988) For more on this issue, see Hochberg (1986).

2. In informal discussions, the subjects reported that the appearance of the luminance changes was nicely consistent with the changes being illumination changes. Under these conditions, the ambiguity of the stimulus was not salient for the observer; there was no feeling that the appearances were unconvincing as illumination changes. The ambiguity is manifested by the changes' being equally convincing as reflectance changes when one was instructed to adopt that set instead.

3. Unlike the equal-reflectance theoretical line, the equal-luminance and equal-luminance-ratio lines are not arbitrary, since they are based on luminances. Their position in our plots depends, of course, on the experimenter's choice of illumination ratios, but only because we chose to plot reflectances as the ordinates. They would be uniquely fixed in a graph of the same data plotted in luminance coordinates.

(Manuscript received March 11, 1992; revision accepted for publication February 26, 1993.) 